\title{
Desarrollo de un Simulador de Oscilación Bioquímica Circadiana
}

DOI: $10.46932 / \mathrm{sfjdv2n1-002}$

Received in: November 1st, 2020

Accepted in: December 30th, 2020

\author{
Jessica Quintero Pérez \\ Doctoranda en Biomecánica y Bioingeniería Aplicadas a la Salud \\ (Universidad de Alcalá, España) \\ PHC. Licenciatura en Fisioterapia-Facultad de Medicina \\ Benemérita Universidad Autónoma de Puebla (BUAP) \\ Área de la Salud. Puebla, Pue. México \\ jessica.quintero@correo.buap.mx \\ Marleni Reyes Monreal \\ Doctoranda en Historia \\ (Instituto de Ciencias Sociales y Humanidades BUAP) \\ PI. Escuela de Artes Plásticas y Audiovisuales (ARPA), BUAP \\ Complejo Cultural Universitario (CCU). Puebla, Pue. México \\ marleni.reyes@correo.buap.mx \\ Arturo Reyes Lazalde \\ Doctorado en Investigación Biomédica Básica (UNAM) \\ PI. Titular Facultad de Ciencias Biológicas, BUAP \\ Ciudad Universitaria. Puebla, Pue. México \\ arturoreyeslazalde@yahoo.com.mx \\ María Eugenia Pérez Bonilla \\ Doctorado en Patología Experimental (CINVESTAV-IPN, Mx) \\ PI. Titular Facultad de Ciencias Biológicas, BUAP \\ Ciudad Universitaria. Puebla, Pue. México \\ maria.perez@correo.buap.mx
}

\section{RESUMEN}

El análisis y comprensión de los procesos fisiológicos de los seres vivos, requiere del desarrollo e interacción académica multidisciplinaria en investigación científica básica, debido a la complejidad y la diversidad biológica de los individuos. Como ejemplo, en este caso se abordan los mecanismos cronobiológicos, cíclicos y circadianos, a nivel molecular. Estos mecanismos, generalmente están basados en un sistema de oscilación periódica diaria ( 24 horas), adaptados a las fases día-noche, regulados exógena, endógena y diferencialmente por los estímulos de luz-oscuridad. Algunas de las funciones biológicas, como el reposo, sueño, actividad, locomoción, metabolismo, apareamiento, desarrollo y envejecimiento, son reguladas por estos sistemas de relojes internos, que operan mediante cambios conformacionales protéicos postraduccionales. El primer mecanismo descrito de autorregulación rítmica gen-proteína fue el modelo PER (period), caracterizado a detalle en el insecto Drosophila melanogaster (mosca de la fruta). En este trabajo se presenta el diseño y desarrollo de un simulador interactivo computacional, que reproduce el ritmo circadiano PER. El simulador llamado "OSCILAR-PER," está basado en el modelo propuesto por Goldbeter (1995), fue desarrollado en lenguaje Visual Basic ${ }^{\circledR}$, versión 5.0, para ambiente Windows ${ }^{\circledR}$, de XP a Windows 10. Las ecuaciones cinéticas que describen los mecanismos se resolvieron por métodos numéricos. Con este programa se 
pueden reproducir los cinco tipos oscilatorios de PER, correspondientes a las curvas de concentración en función del tiempo: del RNA mensajero de la proteína PER, la proteína PER desfosforilada, la PER monofosforilada, la PER bifosforilada y la curva de PER nuclear. Las curvas se pueden simular individual o simultáneamente. El software es una herramienta de apoyo didáctico para la enseñanzaaprendizaje del tema.

Palabras-clave: Software didáctico, modelo de Goldbeter, cinética circadiana PER, cronobiología.

\section{INTRODUCCIÓN}

Los métodos tradicionales de enseñanza-aprendizaje de la biología subcelular, llevan al alumno a pensar que la célula y lo que sucede interiormente: procesos físicos, químicos y moleculares, son estáticos. Las presentaciones PowerPoint ${ }^{\circledR}$, la lectura de textos e incluso artículos especializados, no permiten visualizar la dinámica de los mecanismos que se presentan. Situación que ha motivado la generación de materiales didácticos audiovisuales, como la producción de videos, que se graban durante un experimento y que ahora son parte complementaria importante de algunas publicaciones especializadas. Las diversas problemáticas biomédicas demandan la formación profesional competente de pre y posgrado. Las universidades latinoamericanas han tenido la necesidad de gestionar e implementar la estructura e infraestructura para desarrollar perfiles científicos docentes y estudiantiles que contribuyan al desarrollo nacional (Puente de la Vega, 2020).

Actualmente uno de los graves problemas primarios de salud pública mundial son las enfermedades neurodegenerativas, resultantes del incremento en la expectativa de vida humana (mayores de 80 años) y los estilos de vida. Se ha reportado que la mayoría de los cambios biológicos del crecimiento, desarrollo y envejecimiento están regidos por diversos relojes biológicos ubicados principalmente en el sistema nervioso. Recientemente, algunos de los mecanismos fisiopatológicos de las enfermedades neurodegenerativas se han relacionado con los ritmos de los relojes biológicos circadianos (Emery, 2015). Actualmente, los marcadores moleculares permiten visualizar los eventos subcelulares, por ejemplo: la entrada de calcio a la célula, la oscilación del calcio en un oocito y la distribución protéica. Muchas de las reacciones bioquímicas oscilan periódicamente. Gracias a estas técnicas de registro se han publicado trabajos que reportan datos cuantitativos, que conducen al desarrollo de modelos matemáticos, que reproducen los procesos por computadora. Las bases moleculares de los ritmos circadianos son de gran interés. Por ejemplo, se presume que algunos de ellos están involucrados en diversas patologías, como el cáncer (Sulli, 2019).

Respecto a la trascendencia del tema, se otorgó el Premio Nobel de Fisiología-Medicina 2017, a los Dres. Jeffrey Hall, Michael Young y Michael Rosbash por las aportaciones que condujeron a la comprensión de las bases moleculares de los ritmos circadianos en D. melanogaster; trabajos que 
iniciaron en los años 70 del siglo pasado, con el aislamiento de los mutantes del período (Konopka y Benzer, 1971). Siendo el gen del período (Per) el primer reloj que se aisló y que resultó estar altamente conservado en el reino animal. La experimentación en insectos: pulgón (Acyrthosiphon pisum), mosquito (Anopheles gambiae), abeja (Apis mellifera), mariposa monarca (Danaus plexippus), grillo (Gryllus bimaculatus), cucaracha (Rhyparobia maderae) y escarabajo (Tribolium castaneum), han permitido caracterizar las similitudes y diferencias entre los diversos sistemas cronobiológicos (Beer \& Helfrich-Förster, 2020).

\section{La operatividad de los ritmos circadianos}

Los ciclos circadianos de los relojes biológicos tienen un período aproximado de 24 horas. Los relojes circadianos son mecanismos moleculares que permiten mantener una ritmicidad determinada en una gran variedad de células. Los ritmos circadianos son mecanismos adaptativos fundamentales, que permiten a los organismos regular y controlar la mayor parte de sus funciones biológicas corporales y acoplarlas con las fases de luz-obscuridad características del ciclo día-noche. El ciclo diario de luzoscuridad gobierna los cambios rítmicos fisiológicos de la mayoría de las especies. Adicionalmente, estos ciclos circadianos pueden sincronizarse a señales externas; pero pueden persistir en ausencia de tales señales, regulados por señales internas (endógenas). Diversos estudios han encontrado que los relojes internos consisten en conjuntos de genes y los productos protéicos que codifican y son capaces de regular diversos procesos fisiológicos mediante retroalimentación positiva y negativa. En este trabajo se presenta el diseño y desarrollo de un programa computacional interactivo para la simulación del mecanismo biofísico-molecular de autorregulación del primer reloj circadiano descrito en la neurona de la mosca Drosophila melanogaster, conocido como modelo PER. El simulador está basado en el modelo matemático de Goldbeter (1995).

\section{Modelo biológico de autorregulación del reloj PER en neurona de Drosophila melanogaster}

\section{Mecanismo de retroalimentación negativa que regula la expresión del complejo PER}

A nivel cerebral, el sistema de reloj circadiano de la mosca está conformado por 150-200 neuronas tipo reloj, que se agrupan en siete núcleos, con diez subtipos neuronales diferentes (Shafer, 2006; Helfrich-Föster, 2006).

Muchas de las funciones biológicas de los seres vivos son reguladas por sistemas de relojes que operan molecularmente. En 1984 se reportó el primer gen tipo reloj, llamado "period” (PER), aislado en Drosophila. Consecutivamente se identificaron otros genes tipo reloj circadiano en la misma especie: 
CLK (clock), CRY 1 (cryptochrome 1), CWO (clockwork Orange), CYC (cycle), DBT (double-time), JET (Jetlag), PDF (neuropeptide pigment dispersing factor), PDP1 (par domain protein 1), TIM1 (timeless 1), TIM2 (timeout 2), VRI (vrille) (figura 1).

FIGURA 1. El sistema de reloj circadiano molecular de la Drosophila melanogaster. El mecanismo cronobiológico general está basado en un sistema de oscilación periódica, regulado exógena y endógenamente por los estímulos de luz-oscuridad. Se enlistan los doce genes tipo reloj más estudiados en este modelo, anotados en orden alfabético; así como algunos ejemplos de las funciones que dependen del sistema (basado en Beer \& Helfrich-Förster, 2020).

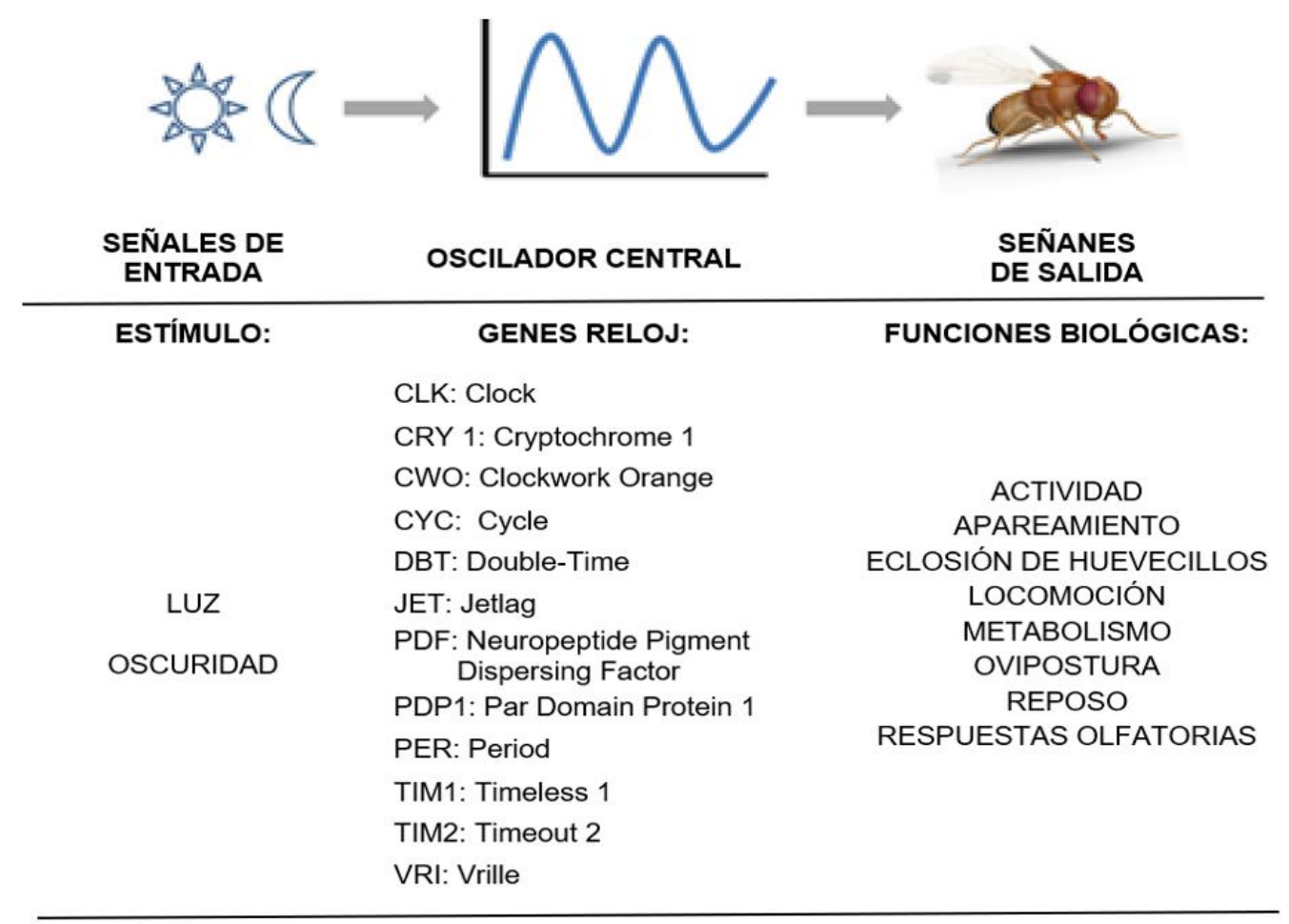

Posteriormente se descubrió que la transcripción del gen PER se induce cuando los niveles del producto, la proteína "period" son reducidos. De modo que Drosophila se convirtió en el primer modelo de autorregulación, mediante la vía de retroalimentación negativa que causaba las oscilaciones repetitivas de la transcripción del gen. En este modelo de experimentación, la expresión molecular de los genes reloj se agrupa en matutina (CLK y CRY), vespertina (PER, TIM, VRI) y nocturna (CYC, DBT), con duración de ocho horas por periodo.

\section{MATERIAL Y MÉTODO}

El simulador está basado en el modelo biofísico de Goldbeter de cinco variables que explica el mecanismo de regulación de la proteína PER, mediante la fosforilación y desfosforilación postraduccional, considera cinco compartimientos básicos: el RNAm de PER (M); la PER desfosforilada (P0); PER monofosforilada (P1); PER bifosforilada (P2) y PER nuclear (PN) (figura 2). 
FIGURA 2. Modelo de oscilación circadiana de la proteína PER en neurona de Drosophila melanogaster. El modelo explica el mecanismo de regulación rítmica de la proteína PER, basado en la modificación postraduccional de PER mediante fosforilación y desfosforilación protéica. Las cinco variables básicas son: M: el RNAm de PER; $\mathrm{P}_{0}$ : PER desfosforilada; $\mathrm{P}_{1}$ : PER monofosforilada; $\mathrm{P}_{2}$ : PER bifosforilada; $\mathrm{P}_{\mathrm{N}}$ : PER nuclear (Goldbeter, 1995).

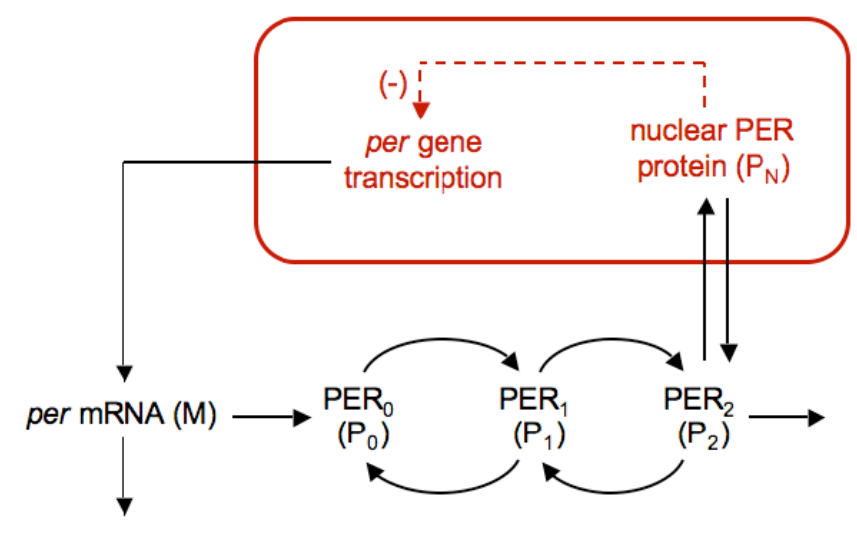

\section{Modelo de oscilación circadiana de Goldbeter de cinco compartimientos:}

\section{Proteína PER de neurona de Drosophila melanogaster}

En la figura 3 se resumen las ecuaciones del modelo de cinco variables de Godbeter (1995), cuya resolución permite simular computacionalmente el mecanismo de regulación oscilatoria circadiana de la proteína PER.

FIGURA 3. Sistema de ecuaciones del Modelo de oscilación circadiana de la proteína PER en neurona de Drosophila melanogaster (Goldbeter,1995).

$$
\begin{aligned}
\text { MENSAJEROPER } & \frac{d M_{P}}{d t}=v_{s} \frac{K_{t}^{n}}{K_{t}^{n}+P_{N}^{n}}-v_{m} \frac{M_{P}}{K_{m}+M_{P}} \\
\text { PER DESFOSFORILADA } & \frac{d P_{0}}{d t}=k_{s} M_{P}-v_{1} \frac{P_{0}}{K_{1}+P_{0}}+v_{2} \frac{P_{1}}{K_{2}+P_{1}} \\
\text { PER MONOFOSFORILADA } & \frac{d P_{1}}{d t}=v_{1} \frac{P_{0}}{K_{1}+P_{0}}-v_{2} \frac{P_{1}}{K_{2}+P_{1}}-v_{3} \frac{P_{1}}{K_{3}+P_{1}}+v_{4} \frac{P_{2}}{K_{4}+P_{2}} \\
\text { PER BIFOSFORILADA } & \frac{d P_{2}}{d t}=v_{3} \frac{P_{1}}{K_{3}+P_{1}}-v_{4} \frac{P_{2}}{K_{4}+P_{2}}-v_{d} \frac{P_{2}}{K_{d}+P_{2}}-k_{1} P_{2}+k_{2} P_{N} \\
\text { PER NUCLEAR } & \frac{d P_{N}}{d t}=k_{1} P_{2}-k_{2} P_{N}
\end{aligned}
$$

Las ecuaciones descritas fueron implementadas en el software de Visual Basic $®$ y resueltas por métodos numéricos. Para establecer los rangos de simulación se predeterminaron los valores de los veinte parámetros cinéticos utilizados en el modelo: vs, ki, n, vm, km, ks, v1, v2, v3, v4, k1, k2, k3, k4, pk1, pk2, vd, kd, vn y kn (Li, 2010). 
Programación del software: El programa se realizó con el lenguaje Visual Basic®, versión 5.0 para ambiente Windows ${ }^{\circledR}$, desde XP a Windows 10, con una resolución de pantalla de 1,366 x 768 pixeles. El programa genera los valores muy cercanos a los reportados (Peschel, 2011).

\section{RESULTADOS}

Se elaboró la segunda versión de un programa computacional, interactivo, autoejecutable directamente, titulado “OSCILAR-PER”. En la figura 4A se muestra la pantalla de presentación y en la 4B el menú principal. El programa se compone de una sección informativa, en la que se incluye la información descriptiva de los conceptos básicos relacionados con el tema. La sección contiene siete opciones: ¿Qué es un ritmo circadiano?, alteraciones en la proteína PER, la retroalimentación negativa, características del gen PER, los comportamientos del gen PER durante un ritmo circadiano, genes que participan en la síntesis del gen PER. Desde cada opción se puede regresar directamente al menú principal (Figura 4B).

FIGURA 4. Pantalla e interfaz del programa. (A) pantalla de presentación del programa. (B) interfaz del menú principal, que consta de ocho botones: siete informativos y el simulador. El programa no tiene un orden predeterminado, el usuario puede navegar a libre elección.

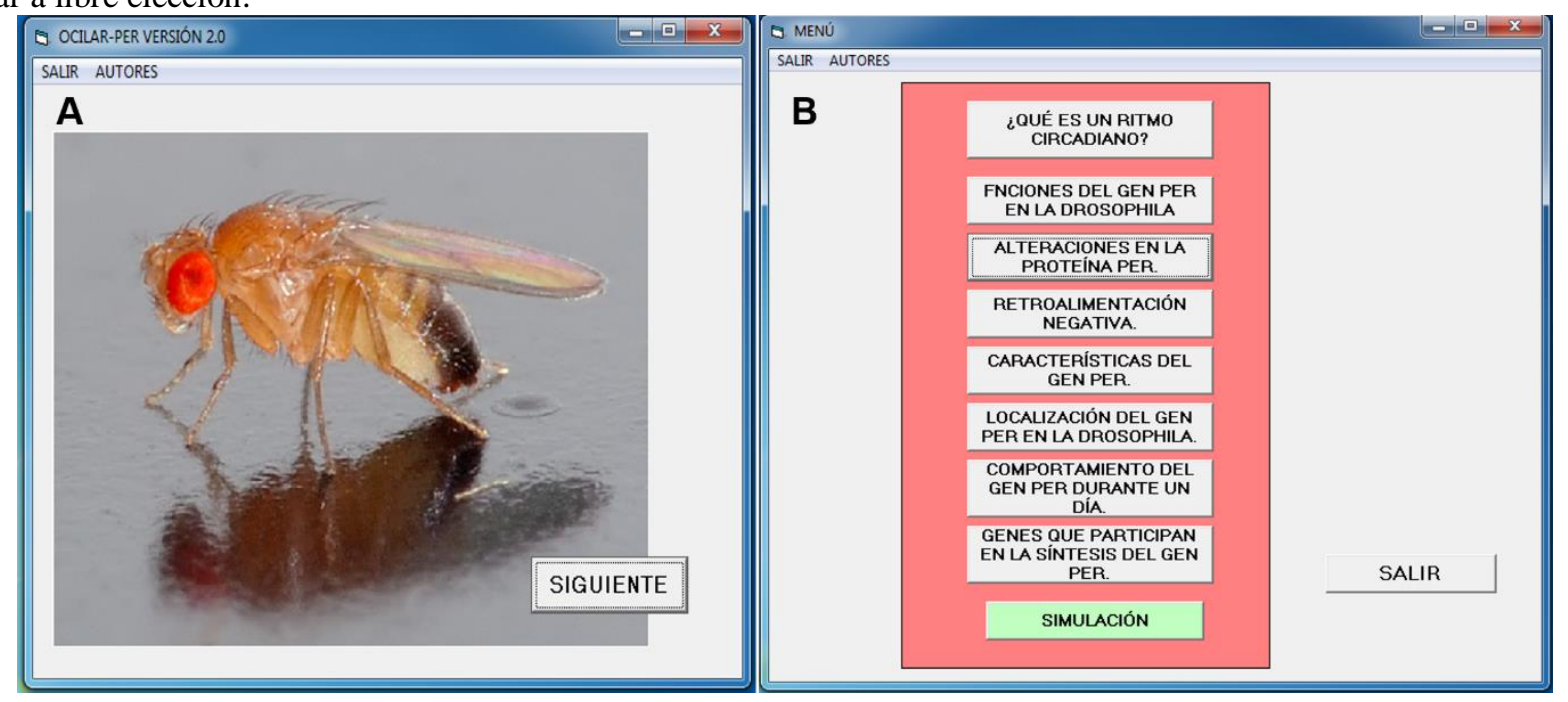

\section{Ejemplo de simulación interactiva}

El simulador describe dos tipos de funciones: las variaciones de concentración de la proteína PER en diversos estados de fosforilación y la concentración del RNAm respectivo. En la interfase de simulación se presenta una pantalla con cinco opciones: el RNA mensajero de PER (M); la proteína PER desfosforilada $\left(\mathrm{P}_{0}\right)$; la PER monofosforilada $\left(\mathrm{P}_{1}\right)$; la curva de PER bifosforilada $\left(\mathrm{P}_{2}\right)$ y la curva de PER nuclear $\left(\mathrm{P}_{\mathrm{N}}\right)$ (figura 5). En todos los casos el usuario puede observar el comportamiento oscilatorio del ritmo circadiano de la proteína PER en un periodo aproximado de cuatro días y medio. 
FIGURA 5. Interfaz del simulador con las cinco opciones de graficado: el RNA mensajero de PER (M); la proteína PER desfosforilada (P0); la PER monofosforilada (P1); la curva de PER bifosforilada (P2) y la curva de PER nuclear (PN). En este ejemplo se muestra en azul la curva del RNAm.

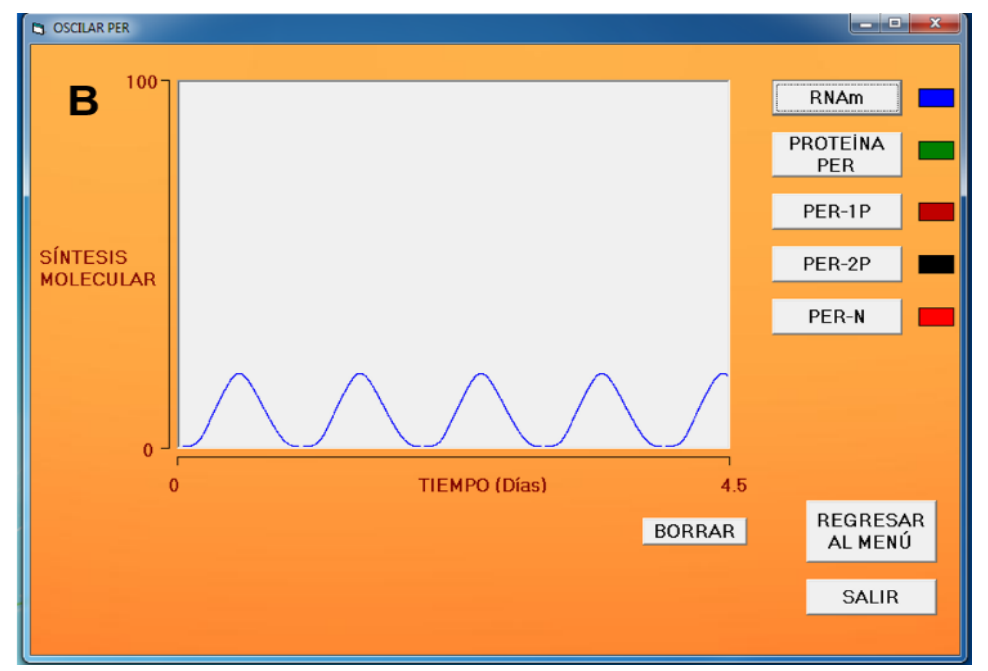

El comportamiento de los ritmos circadianos se puede analizar a través de gráficas sinusoidales, que describen su naturaleza periódica y oscilante por medio de los siguientes parámetros: Amplitud (diferencia entre el valor máximo de la variable y el menor); Periodo (tiempo que dura una oscilación completa); Valor medio o mesor (media de los valores que toma un parámetro durante un ciclo); Fase (tiempo en que se alcanza el valor máximo de un periodo) y Frecuencia (número de ciclos por unidad de tiempo). Las curvas se pueden visualizar individualmente o bien simultáneamente. En la figura 6A se muestra un ejemplo de doble graficado y en la 6B las cinco curvas simultáneamente.

FIGURA 6. Pantalla e interfaz del programa. (A) en color azul el mensajero de PER (M) y en verde la proteína PER desfosforilada (P0). (B) en azul el mensajero de PER (M); en verde la PER desfosforilada (P0); en rojo la PER monofosforilada (P1); en morado la curva de PER bifosforilada (P2) y en color naranja la curva de PER nuclear (PN).

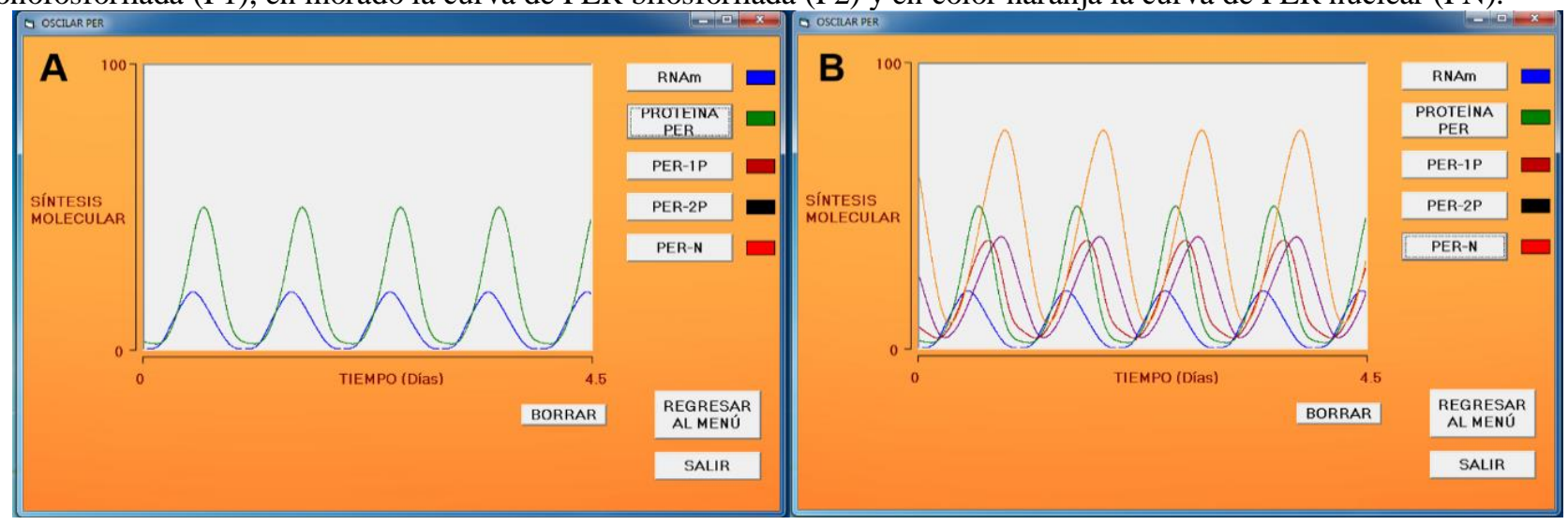




\section{CONCLUSIONES}

El programa "OSCILAR-PER” es una herramienta computacional, didáctica, de fácil manejo. El usuario tiene la facilidad de acceder de forma rápida a cada apartado, con la posibilidad de cambiar de interfaz fácilmente para interactuar con cada opción del simulador.

Con el módulo informativo el usuario puede adquirir información directa, de forma general y clara sobre los procesos relacionados y los factores que intervienen e interactúan para dar lugar a los cambios conformacionales proteicos de PER.

Con el módulo de simulación se pueden reproducir los cinco tipos oscilatorios PER correspondientes a las curvas de concentración en función del tiempo: el RNA mensajero de la proteína PER (M); la proteína PER desfosforilada $\left(\mathrm{P}_{0}\right)$; la PER monofosforilada $\left(\mathrm{P}_{1}\right)$; la PER bifosforilada $\left(\mathrm{P}_{2}\right)$ y la curva de PER nuclear $\left(\mathrm{P}_{\mathrm{N}}\right)$. Las curvas se pueden simular individual o simultáneamente. 


\section{REFERENCIAS}

Beer, K. \& Helfrich-Förster, C. (2020). Model and non-model insects in chronobiology. Front. Behav. Neurosc. 14(601676): 1-23. https://doi.org/10.3389/fnbeh.2020.601676.

Emery, P. (2015). Connecting circadian genes to neurodegenerative pathways in fruit flies. PLOS Genet. 11(6), e1005266. https://doi.org/10.1371/journal.pgen.1005266.

Glossop, N. R. (2011). Circadian timekeeping in Drosophila melanogaster and Mus musculus. Essays Biochem. 49(1): 19-35. https://doi.org/10.1042/bse0490019.

Goldbeter, A. (1995). A model for circadian oscillations in the Drosophila period protein (PER). Proc. Biol. Sci. 261(1362): 319-324. https://doi.org/10.1098/rspb.1995.0153.

Helfrich-Förster, C., Shafer, O. T., Wülbeck, C., Grieshaber, E., Rieger, D. \& Taghert, P. (2006). Development and morphology of the clock-gene-expressing lateral neurons of Drosophila melanogaster. J. Comp. Neurol. 500(1): 47-70. https://doi.org/10.1002/cne.21146.

Konopka, R. J. \& Benzer, S. (1971). Clock mutants of Drosophila melanogaster. Proc. Natl. Acad. Sci. USA. 68(9): 2112-2116. https://doi.org/10.1073/pnas.68.9.2112.

Li, C., Nagasaki, M., Saito, A. \& Miyano, S. (2010). Time-dependent structural transformation analysis to high-level Petri net model with active state transition diagram. BMC. Syst. Biol. 4(39): 1-14. https://doi.org/10.1186/1752-0509-4-39.

Peschel, N. \& Helfrich-Förster, C. (2011). Setting the clock - by nature: circadian rhythm in the fruitfly Drosophila melanogaster. FEBS Lett. https://doi.org/10.1016/j.febslet.2011.02.028.

Puente de la Vega V. (2020). Gestión de la Investigación en las Universidades Públicas del Perú: avances desde la Implementación de la Ley Universitaria 30220. SFJD 1(4): 268-286. ISSN 2675-5459. https://doi.org/10.46932/sfjdv1n4-011.

Shafer, O. T., Helfrich-Förster, C., Renn, S. C. P. \& Taghert, P. H. (2006). Reevaluation of Drosophila melanogaster's neuronal circadian pacemakers reveals new neuronal classes. J. Comp. Neurol. 498(2): 180-193. https://doi.org/10.1002/cne.21021.

Sulli, G., Lam, M. \& Panda, S. (2019). Interplay between circadian clock and cancer: new frontiers for cancer treatment. Trends Cancer. 5(8): 475-494. https://doi: 10.1016/j.trecan.2019.07.002 